\title{
Determination of Aluminum in Copper-Aluminum Alloy Using Gamma-Ray Spectrometry
}

\author{
A.H. El-Kateb, R. A. M .Rizk and A. M. Abdul-Kader \\ Physics Department, Faculty of Science, Helwan Univ., \\ Ain Helwan, Cairo, Egypt
}

\begin{abstract}
In this work, a nuclear gauge, based on single and dual energy $\gamma$-ray transmissions, has been developed and used to determine the concentrations of the aluminum component in copper-aluminum alloy. Disk foils of $7.07 \mathrm{~cm}^{2}$ area and thicknesses ranging from 0.48 to $0.834 \mathrm{~cm}$ have been prepared from the alloy to be used as targets for the $\gamma$-ray photons. Criteria for the best choice of the suitable photon energies for both single and dual energy $\gamma$-ray transmissions have been investigated. In this work, aluminum concentration values with an accuracy of $98.53 \%$ and $99.09 \%$ have been obtained by using both single and dual $\gamma$-ray energy transmission techniques, respectively. A comparison of the present results with those from the conventional chemical analysis indicates a fair agreement. A criterion for the best choice of the suitable $\gamma$-ray transmission technique for the aluminum determination has been investigated.
\end{abstract}




\section{Introduction}

Gamma-ray absorptiometry is widely used in non-destructive characterization of opaque materials [1-7]. Many other methods such as X-ray fluorescence spectrometry [8], prompt $\gamma$-ray neutron activation analysis [9], chemical analysis, and instrumental neutron activation analysis [10] could b used for material characterization. The main advantages of gamma-ray absorptiometry as an analytical tool are the following [2,3,7]:

a) its simplicity for handling, instrumentation, and processing,

b) it can provide representative analysis without the need of sample preparation procedures,

c) it can give an early indication of the mineral quality by allowing direct determination of concentration of sensitive elements and the derivation of important economic parameters, such as ash content value in samples, in conveyor belts and in ores, and

d) it is non-destructive, accurate, economic, and low cost analytical tool.

In this work, single and dual energy $\gamma$-ray transmission techniques are used to determine the aluminum component in copper-aluminum alloy. Criteria for the best choice of the suitable photon energies for both single and dual energy $\gamma$-ray transmissions have been investigated. In this work, aluminum concentration values with an accuracy of $98.53 \%$ and $99.09 \%$ (100 - fraction error) have been obtained by using both single and dual $\gamma$-ray energy transmission techniques, respectively. A comparison of the resent results with those obtained from the conventional chemical analysis indicates a fair agreement. A criterion for the best choice of the suitable $\gamma$-ray transmission technique for the aluminum determination has been investigated. Preliminary results show that the suggested technique can be successfully applied in composition characterization of materials to give a rapid and economic determination of the concentration of certain elemental components.

\section{Gamma-Ray Transmission Techniques}

The 1980s have seen the development and the widespread use of the industrial application of a new generation of nuclear gauges [11]. Among the measurement techniques used in this new generation of nuclear gauges are the following:

\section{Single Energy Gamma-Ray Transmission Technique (SET):}

The intensity lo of a collimated $\gamma$-ray beam will be attenuated to an intensity 1 after it has passing through an absorbing material mixture of mass thickness $\mathrm{x}$ according to the following general absorption law [12]: 


$$
I=I_{o} e^{-\mu_{t} x}
$$

where $\mu_{\mathrm{t}}$ is the total mass absorption coefficient which can be given by

$$
\mu_{t}=\sum_{i} \mu_{i} C_{i}
$$

with

$$
\sum_{i} C_{i}=1
$$

where $\mu_{\mathrm{i}}$ and $\mathrm{C}_{\mathrm{i}}$ are the mass absorption coefficient and the weight fraction of the $i^{\text {th }}$ component in the material mixture, respectively.

In the SET technique [2,7], an unknown concentration of a material component is determined by measuring the intensities of a narrow beam of low or high-energy $\gamma$-ray that is transmitted through an absorbing material of mass thickness $\mathrm{x}\left(\mathrm{gm} / \mathrm{cm}^{2}\right)$. In the case that case that the absorbing material is a binary alloy such as $\mathrm{Cu}-\mathrm{Al}$ alloy, equations 1,2 and 3 can be combined to give an expression for aluminum concentration, $\mathrm{C}_{\mathrm{Al}}$, in the alloy as follows :

$$
\mathrm{c}_{\mathrm{Al}}=\left\{\ln \left(\frac{\mathrm{I}_{\mathrm{o}}}{\mathrm{I}}\right) / \mathrm{x}-\boldsymbol{\mu}_{\mathrm{Cu}}\right\} /\left(\mu_{\mathrm{Al}}-\mu_{\mathrm{Cu}}\right)
$$

where $\mu_{\mathrm{Al}}$ and $\mu_{\mathrm{Cu}}$ are the mass absorption coefficients of aluminum and copper, respectively. Eqn. 4 represents a straight-line relation between $\mathrm{C}_{\mathrm{Al}}$ and $\left\{\frac{1}{x} \ln \left(\frac{I_{o}}{I}\right)\right\}$

\section{Dual Energy Gamma-Ray Transmission (DET) Technique :}

In the DET technique [2,7], the content of a specific component is determined by combining measurements of intensities of narrow beams of low and high-energy $\gamma$-rays transmitted through the sample. According to the formula derived by Watt and Steffner [12], the percentage concentration of aluminum in copper -aluminum alloy is determined by using the following expression :

$$
C_{A l}=\left(\mu_{C u}^{\prime \prime}-R \cdot \mu_{C u}^{\prime}\right) /\left\{R\left(\mu_{A l}^{\prime}-\mu_{C u}^{\prime}\right)+\left(\mu_{C u}^{\prime \prime}-\mu^{\prime \prime}{ }_{A l}\right)\right\}
$$

The superscripts ' and " refer to the lower and higher $\gamma$-ray energies, respectively. $\mathrm{R}$ is the ratio of the mass absorption coefficients for the material sample at the higher and lower $\gamma$-ray energies; it is given by 


$$
R=\ln \left(I_{o} / I\right)^{\prime \prime} / \ln \left(I_{o} / I\right)^{\prime}
$$

$\mu{ }_{A l} \approx \mu{ }_{C u}\left(\mu{ }_{A l}=0.097\right.$ and $\left.\mu{ }_{C u}=0.101 \mathrm{~cm}^{2} / \mathrm{g}\right)$, therefore the term $\left(\mu{ }_{C u}\right.$ $\left.\mu{ }_{A l}\right)$ in Eqn. (5) should be omitted. Accordingly, Eqn. (5) should be rewiitten in the following from:

$$
C_{A l}=\left\{\mu_{C u}^{\prime \prime} / R\left(\mu_{A l}^{\prime}-\mu_{C u}^{\prime}\right)\right\}-\mu_{C u}^{\prime} /\left(\mu_{A l}^{\prime}-\mu_{C u}^{\prime}\right)
$$

which represents a straight line between $\mathrm{C}_{\mathrm{Al}}$ and $1 / \mathrm{R}$.

\section{Error Analysis}

In SET technique, the errors in the measured aluminum concentration, $C_{A l}$ may be due to errors in the measured $\gamma$-ray intensities, material density $(\rho)$ and the linear thickness of the target $(\mathrm{d}) ; \mathrm{x}=\rho \mathrm{d}$. The error in the concentration of the determined component can be estimated by the partial differentiation of Eqn.(4).

$$
\delta C_{A l}=\frac{\left(\left(I \delta_{I_{o}}-I_{0} \delta I\right) /\left(I I_{o}\right)-\frac{1}{d \rho}(d \delta \rho+\rho \delta d) \ln \left(I_{o} / I\right)\right)}{d \rho\left(\mu_{A l}-\mu_{C u}\right)}
$$

The relative error in $C_{A l}$ has been estimated to be in the order of magnitude of $1.5 \%$.

In the present study, the errors in $\mathrm{d}$ and $\rho$ are negligible, so that $\delta \mathrm{d}$ and $\delta \rho$ may be omitted in Eqn. (8) to be in the from :

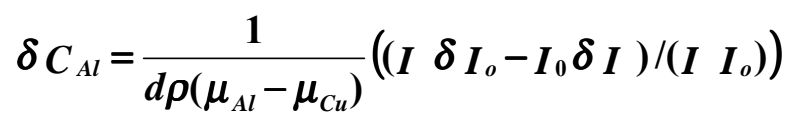

or

$$
\delta C_{A l}=\frac{\delta \mu_{t}}{\left(\mu_{A l}-\mu_{C u}\right)}
$$

where $\delta \mu_{\mathrm{t}}$ is the error in the total mass absorption coefficient $\mu_{\mathrm{t}}$ and it is given by:

$$
\delta \mu_{t}=\frac{1}{d p}\left(\frac{I \delta I_{o}-I_{o} \delta I}{I I_{o}}\right)
$$

The relative error in $\mu_{\mathrm{t}}$ has been estimated; it was found to be $\leq 1.49 \%$. 
Eqn.(10) can be reformulated on the following form to give the sensitivity of $C_{A l}$ to the relative error in $\mu_{\mathrm{t}}$.

$$
\delta C_{A l} /\left(\delta \mu_{t} / \mu_{t}\right)=\frac{\mu_{t}}{\left(\mu_{A l}-\mu_{C u}\right)}
$$

In DET technique, the relative errors in the measured aluminum concentration, $C_{A l}$, are due to the errors in the experimental measurements of R, which in turn depends on the measured intensity. Partial differntiontion of Eqn.(6) gives the error in $\mathrm{R}$ as follows:

$$
\delta R=\frac{1}{\ln \left(\zeta_{o} / I\right)^{\prime}} \sqrt{\left(\frac{\left(I^{\prime \prime} \delta I_{o}^{\prime \prime}-I_{0}^{\prime \prime} \delta I^{\prime \prime}\right)}{I^{\prime \prime} \cdot I_{o}^{\prime \prime}}\right)^{2}+R^{2}\left(\frac{\left(I^{\prime} \delta I_{o}^{\prime}-I_{o}^{\prime} \delta I^{\prime}\right)}{\left(I^{\prime} \cdot I_{o}^{\prime}\right)}\right)^{2}}
$$

The relative error in $\mathrm{R}$ has been estimated to be in the order of the magnetude of $3 \%$. Partial differentiation of Eqn. (5) gives the error of the aluminum concentration, which is due to the inaccuracy in the measurement of $\mathrm{R}$ as follows:

$$
\delta c_{A l}=\frac{\left\{\left(\mu_{C u}^{\prime}+C_{A l}\left(\mu_{A l}^{\prime}-\mu_{C u}^{\prime}\right)\right\}-\delta R\right.}{\left\{R\left(\mu_{A l}^{\prime}-\mu_{C u}^{\prime}\right)+\left(\mu_{C u}^{\prime \prime}-\mu^{\prime \prime}{ }_{A l}\right)\right.} .
$$

The relative error in $\mathrm{C}_{\mathrm{Al}}$ was estimated to be of the order of magnitude of $0.9 \%$. the sensitivity of $\mathrm{C}_{\mathrm{Al}}$ to the relative error in $\mathrm{R}$ can be given by the reformulation of Eqn. (13) to be in the form:

$$
\delta C_{A l} /(\delta \boldsymbol{R} / R)=\frac{-\left(\left(\mu_{C u}^{\prime}+c_{A l}\left(\mu_{A l}^{\prime}-\mu_{C u}^{\prime}\right)\right) R\right.}{\left(R\left(\mu_{A l}^{\prime}-\mu_{C u}^{\prime}\right)+\left(\mu_{C u}^{\prime \prime}-\mu_{A l}^{\prime \prime}\right)\right)}
$$

\section{Experimental}

\section{Target foils}

Disk foils of $7.07 \mathrm{Cm}^{2}$ area and thickness ranging from 0.48 to $0.834 \mathrm{Cm}$ have been prepared from the $\mathrm{Cu}-\mathrm{Al}$ alloy to be used as targets with different $\mathrm{Al}$ concentration for the $\gamma$-ray photons. In this work, it was found that when the $\mathrm{Al}$ concentration exceeds $31 \%$, the prepared sample would be distructed. 


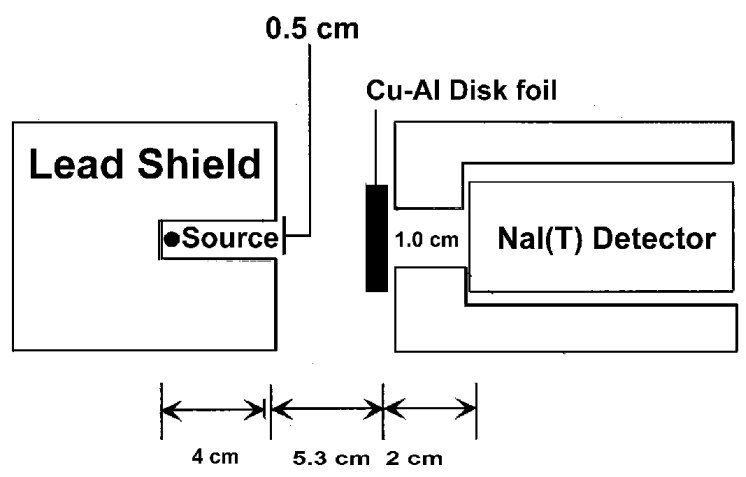

Fig. (1) Experimental set up and arrangement

\section{Experimental Set-Up}

The experimental setup consists mainly of a CANBERRA 3 " x 3 " Nal (Tl) crystal (model 2007P) coupled to a NUCLEUS PCA 8192 multichannel analyzer. The whole experimental setup is shown in Fig. (1). The samples were prepared in the form of cylinderical discs of $3 \mathrm{~cm}$ diameter and thicknesses in the range of 0.39 to $0.83 \mathrm{~cm}$ at 0.081 and $0.356 \mathrm{MeV} \gamma$-ray energies. ${ }^{133} \mathrm{Ba}$ as a $\gamma$-ray source of stren- gth $\cong 4 \mu \mathrm{Ci}$ "AMERSHAM" was used in the experiment.

\section{Experimental precautions}

Using a high accuracy digital balance will help in minimizing the errors in measurements. The background and peak centeroids were checked carefully during the $\gamma$-ray spectrum measurements. The $\gamma$-ray beam was checked using a laser beam (HELIUM-NEON/0.95 mW laser source, SPECTRA PHYSICS typemodel 155). Effects due to temperature, applied voltage, and detector efficiency were minimized by stabilizing the voltage source and by air conditioning the laboratory. In addition to these necessary precautions, the photo-peak windows that gave experimental mass absorption coefficient values for $\mathrm{Cu}$ and $\mathrm{Al}$ in fair agreement with the theory were selected to determine the total photo-peak area.

\section{Results and Discussion}

From Eqn. (4) it's clear that if $\mu_{\mathrm{Al}}$ approaches $\mu_{\mathrm{Cu}}$ the dominator in the equation tends to zero and $\mathrm{C}_{\mathrm{Al}}$ will tend to infinity. In such a case it will not be possible to construct an analytical calibration curve in its normal meaning. For this reason, the most important criterion in this work is that the absorption coefficient of one of the material components should be much smaller than the absorption coefficient of the other one. In such a case, the total absorption is mainly due to this absorbing component,and a calibration curve using its variation is possible.Table (1) 
gives the values of the absorption coefficients $\mu_{\mathrm{Al}}$ and $\mu_{\mathrm{Cu}}$ at 81 and $356 \mathrm{keV} \gamma$-ray energies [13].

From Table (1), it's clear that the mass absorption coefficient of $\mathrm{Cu}$ is nearly 4 times the mass absorption coefficient of $\mathrm{Al}$, which satisfies the previously mentioned criterion.

Table (1): The mass absorption coefficients of $\mathrm{Cu}$ and $\mathrm{Al}$ components in the $\mathrm{Cu}-\mathrm{Al}$ alloy at 81 and $356 \mathrm{keV} \gamma$-ray energies

\begin{tabular}{|c|c|c|}
\hline \multirow{2}{*}{$\begin{array}{l}\text { Mass absorption coefficients of the } \mathrm{Cu}- \\
\mathrm{Al} \text { components }\left(\mathrm{cm}^{2} / \mathrm{gm}\right)\end{array}$} & \multicolumn{2}{|c|}{$\gamma$-ray energies (keV) } \\
\hline & 81 & 356 \\
\hline$\mu_{\mathrm{Cu}}$ & 0.74 & 0.101 \\
\hline$\mu_{\mathrm{Al}}$ & 0.20 & 0.097 \\
\hline
\end{tabular}

The value of the $\gamma$-ray energy that satisfy the above mentioned criterion has been investigated. Fig. (2) shows the variation of the total mass absorption coefficient, $\mu \mathrm{t}$, with the percentage concentration of $\mathrm{Al}, \mathrm{C}_{\mathrm{Al}}$, at the $\gamma$-ray energies 81 and $356 \mathrm{keV}$. From the figure, it can be seen that the $\gamma$-ray absorption in the alloy is clearly sensitive to the variation in the $\mathrm{Al}$ concentration at $81 \mathrm{keV} \gamma$-ray energy while at the other energy the response is not remarkable.

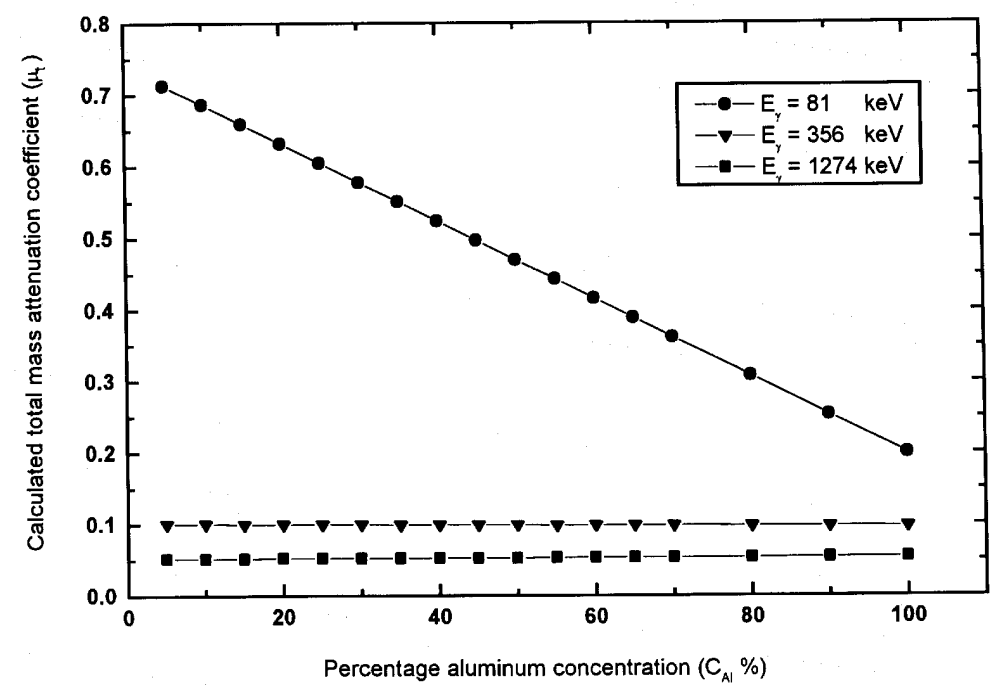

Fig. (2) Variation of both calculated and measure total mass absorption coefficients with $\mathrm{Al}$ concentration at 81 and $356 \mathrm{keV} \gamma$-ray energies.

Equation (11) has been used to clarify the correlation between the variation in the percentage $\mathrm{Al}$ concentration $\left(\delta \mathrm{C}_{\mathrm{Al}}\right)$ and the variation in the relative error $(\delta \mu \mathrm{t} / \mu \mathrm{t})$ in the total mass absorption coefficient. Fig. (3) chose the dependence of the variations $\left(\delta \mathrm{C}_{\mathrm{Al}}\right)$ in the percentage $\mathrm{Al}$ concentration on the 
normalized relative error $(\delta \mu \mathrm{t} / \mu \mathrm{t})$. A percentage error of one percent in $\mu \mathrm{t}$ corresponds to variations of $0.01,0.26$ and 0.23 in the percentage $\mathrm{Al}$ concentration at the $\gamma$-ray energies 81,356 , and $1274 \mathrm{keV}$, respectively. This indicates that the variation of the percentage $\mathrm{Al}$ concentration with the percentage error in $\mu \mathrm{t}$ at the photopeak line $81 \mathrm{keV}$ is clearly smaller than that at the other two-photopeak lines. From the above discussion, it is conclusively evident that the line $81 \mathrm{keV}$ is more preferable to be used as a SET gauge for Al determination in $\mathrm{Cu}-\mathrm{Al}$ alloy. A calibration curve between $\mathrm{C}_{\mathrm{Al}} \%$ and $1 / \mathrm{x} \ln \left(\mathrm{I}_{\alpha} / \mathrm{I}\right)$ shows that, the $\mathrm{Al}$ concentration should not exceed $31 \%$ because as mentioned before when the $\mathrm{Al}$ concentration exceeds $31 \%$ the target foil will be destructed.

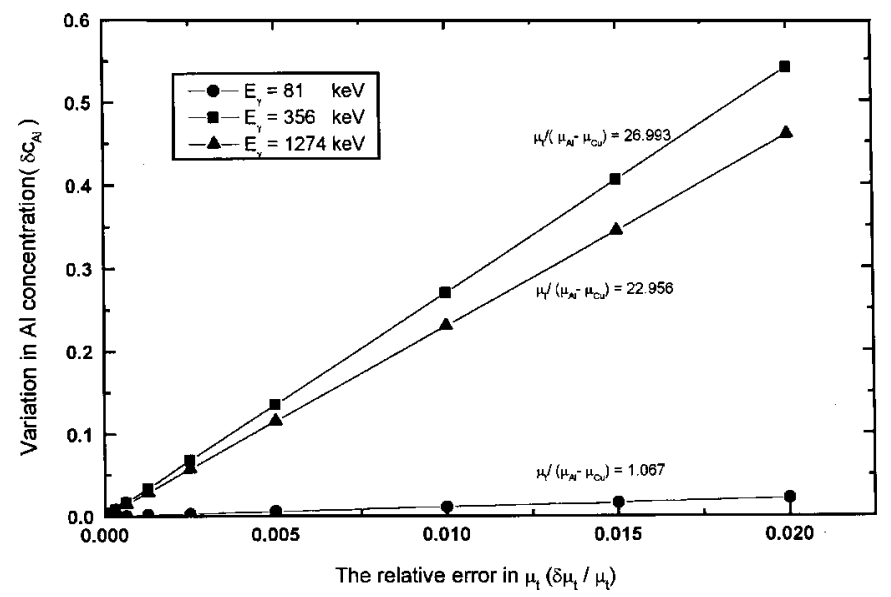

Fig. (3) Effect of the error in the total mass absorption coefficient on the variation in aluminum concentration in an alloy with $69.64 \% \mathrm{Cu}$ and $30.36 \% \mathrm{Al}$ at 81,356 and $127 \mathrm{keV} \gamma$-ray energies

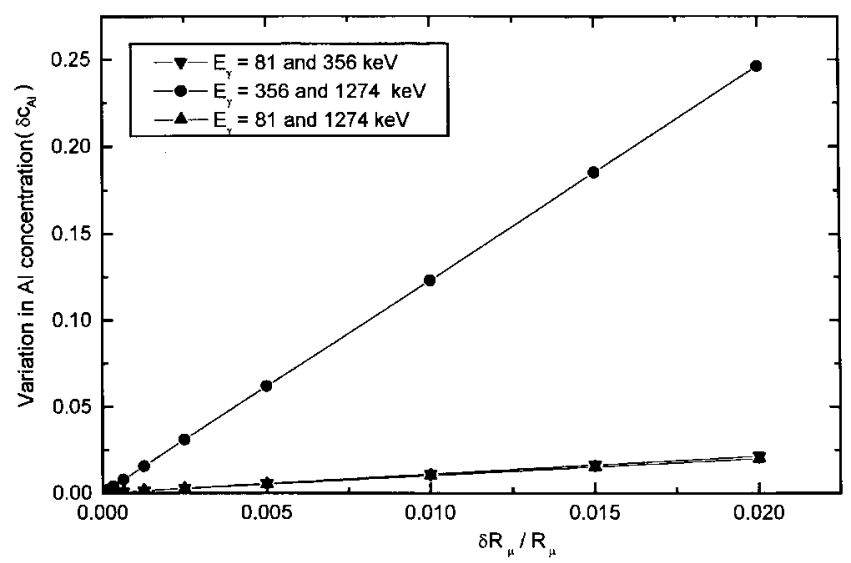

Fig. (4) Effect of the error in $\mathrm{R}_{\mu}$ on the variation in $\mathrm{Al}$ concentration in an alloy of $69.64 \%$ and $30.36 \%$ at three different energy couples. 
In order to determine the suitability of a $\gamma$-ray energy couple to be used as a dual $\gamma$-ray energy beam, Eqn. (14) has been applied to calculate $\delta \mathrm{C}_{\mathrm{AL}}$ for a sample of known concentrations in terms of $\delta R / R$. Fig. (4) shows $\delta C_{A l}$ versus $\delta R / R$ as obtained from Eqn. (14). It is obvious that the relation is a straight line of a slope, which is dependent on the value of $\mu$ and $R$. It could be easily noted that there is no an observable difference between the energy couples $(81,356 \mathrm{keV})$ and $(81,1274 \mathrm{keV})$, while for the energy couple $(356,1274 \mathrm{keV}) \delta \mathrm{C}_{\mathrm{Al}}$ shows a large sensitivity to $\delta \mathrm{R} / \mathrm{R}$. a percentage error of $1 \%$ in $\delta \mathrm{R} / \mathrm{R}$ corresponds to the variations of $0.011,0.0101$ and 0.123 in the $\mathrm{Al}$ concentration percentage $\left(\mathrm{C}_{\mathrm{Al}}\right)$ at the energy couples $(81,356 \mathrm{keV}),(81,1274 \mathrm{keV})$ and $(356,1274$ $\mathrm{keV}$ ), respectively. So, it could be concluded that Fig. (4) does not recommend the energy couple $(356,1274 \mathrm{keV})$ as a partner in the present proposed dual energy transmission technique. Accordingly, the energy couple $(356,1274 \mathrm{keV})$ should be excluded in this work. More over, the experimental setup is based on the using of narrow beam geometry, so that Compton continuum is not effective. The energy couple of the two photolines 81 and $356 \mathrm{keV}$ is emitted from a compact radioactive source ${ }^{133} \mathrm{Ba}$, therefore a good beam geometry with a good source positioning and a beam alignment could be attained. The choice of the energy couple for DET technique should be governed by all these previous criteria. Accordingly, the energy couple $(81,356 \mathrm{keV})$ is chosen as a dual energy beam to be used in the present work. Although, Fig. (2) demonstrates that the DET technique is of no value, since at the $356 \mathrm{keV}$ energy there is no response, and the use of this technique adds nothing new to the SET technique, the DET technique has the advantage of its independence on the target thickness. This enables us to avoid any errors due to thickness measurements, which by role reflects on accurate determination of $\mathrm{Al}$ concentration. According to Eqn. (8) a calibration curves between $\mathrm{C}_{\mathrm{Al}} \%$ and $1 / \mathrm{R}$ which is equal to $\ln \left(\mathrm{I}_{0} / \mathrm{I}\right)^{\prime} / \ln \left(\mathrm{I}_{0} / \mathrm{I}\right)^{\prime \prime}$ has been invesitgated.

The accuracy of the present $\mathrm{Al}$ determination by using both single and dual $\gamma \gamma$ ray energy transmission techniques has been tested by analyzing the measured samples using the conventional chemicals analysis technique. Average relative errors of the order of magnitudes $\pm 1.69 \%$ and $\pm 1.25 \%$ have been assigned to the results from both single and dual $\gamma$-ray energy transmission techniques, respectively. In Figs. $(7,8)$, the percentage aluminum concentration values from the SET gauge $(81 \mathrm{keV})$ and the DET gauge (81, $356 \mathrm{keV}$ ), respectively, are compared with those values obtained from conventional chemical analysis. Both figures show linear relationships between the aluminum concentration values as obtained from the $\gamma$-ray absorption measurements and those obtained from chemical analysis. A linear regression has been made for the data in both figures. Linearity with r.m.s estimating error of \pm 0.4 and $\pm 0 . .37$ for both figures, respectively, has been attained.

The errors in the mass thickness $x$ and density $\rho$ in addition to the errors in $I_{0}$ and I are the main sources of the errors in the SET gauge. On the other hand $I_{0}^{\prime}, I^{\prime}, I_{0}^{\prime \prime}$ and $I^{\prime \prime}$ are the main sources of the errors in the DET gauge. As the conclusion, the values of these errors stand behind the decision which gauge (SET or DET) can be used for the determination of $\mathrm{Al}$ concentration in $\mathrm{Cu}-\mathrm{Al}$ alloy. In some details, if $\mathrm{x}$ is large and $\rho$ is also large, the DET gauge is recommended. While, if $I_{0}^{\prime}, I^{\prime}, I_{0}^{\prime \prime}$ and I" values are not accurately enough due to, e.g., Compton continuum and low $\gamma$-ray counting rates and if 
there is no significant error in the thickness $\mathrm{x}$, the SET gauge is the best technique and strongly recommended.

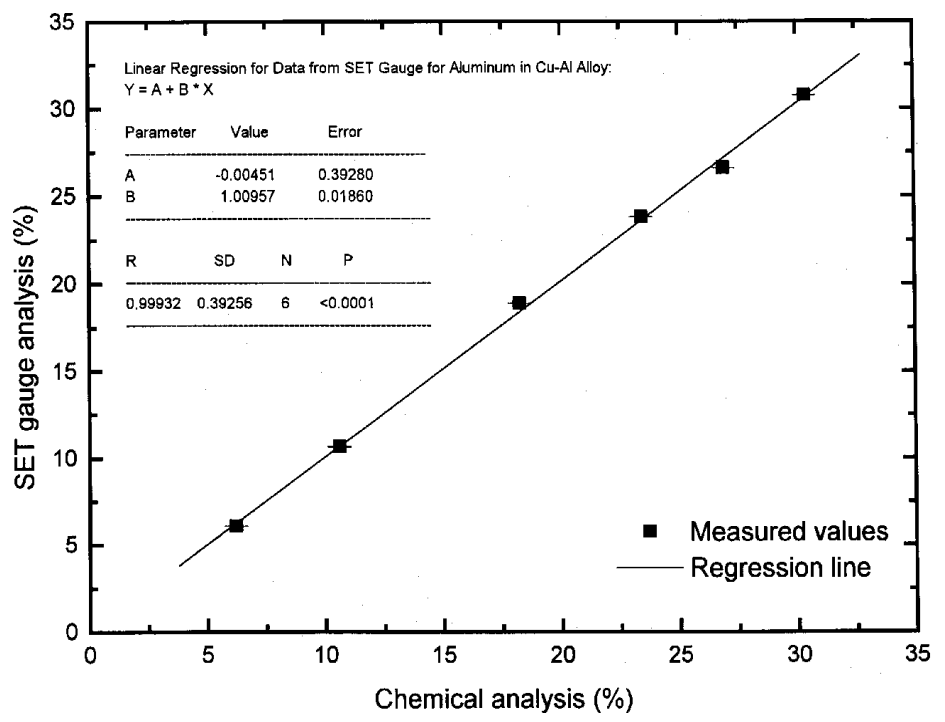

Fig. (5) A comparison between results from the SET gauge for $\mathrm{Al}$ in $\mathrm{Cu}-\mathrm{Al}$ alloy and the those results from chemical analysis.

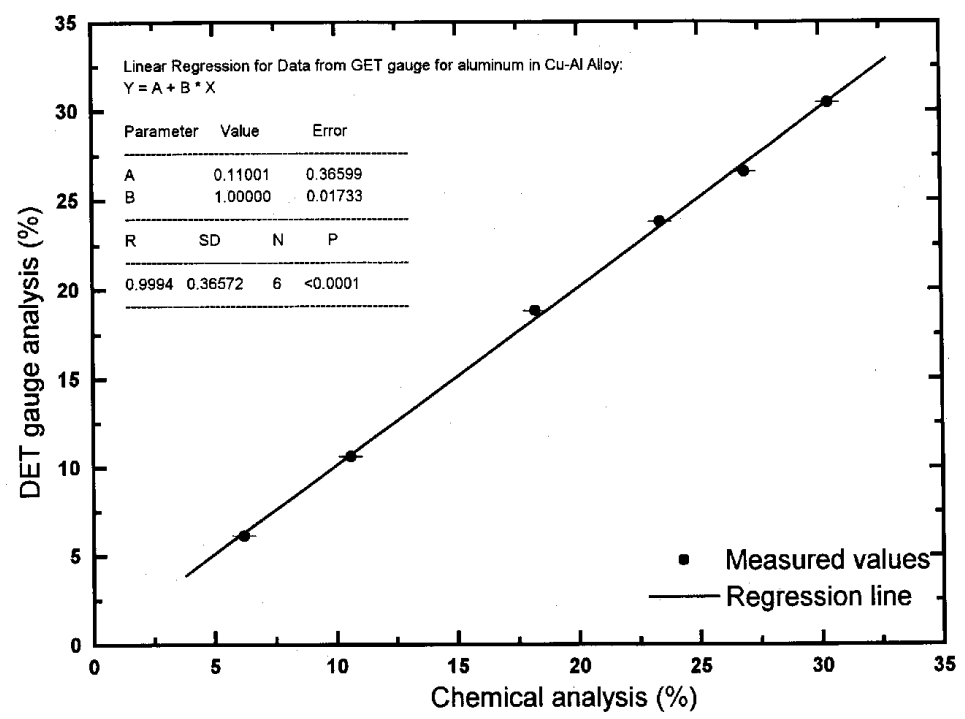

Fig. (6) A comparison between results from the DET gauge for $\mathrm{Al}$ in $\mathrm{Cu}-$ $\mathrm{Al}$ alloy and those results from chemical analysis. 
Table (2) : Aluminum concentration values in Copper-Aluminum alloy as obtained from chemical analysis and $\gamma$-ray transmission techniques.

\begin{tabular}{|c|c|c|}
\hline $\begin{array}{c}\text { Chemical analysis \% } \\
\text { (standards) }\end{array}$ & $\begin{array}{c}\text { SET gauge analysis \% } \\
\mathbf{( 8 1} \mathbf{~ k e V )}\end{array}$ & $\begin{array}{c}\text { DET gauge analysis \% } \\
\mathbf{( 8 1 , 3 5 6 ~ k e V )}\end{array}$ \\
\hline 6.2 & $6.07 \pm 0.089$ & $6.13 \pm 0.055$ \\
\hline 10.6 & $10.65 \pm 0.081$ & $10.62 \pm 0.051$ \\
\hline 18.23 & $18.89 \pm 0.086$ & $18.81 \pm 0.050$ \\
\hline 23.4 & $23.81 \pm 0.097$ & $23.79 \pm 0.058$ \\
\hline 26.9 & $26.61 \pm 0.05$ & $26.57 \pm 0.024$ \\
\hline 30.36 & $30.74 \pm 0.074$ & $30.43 \pm 0.034$ \\
\hline
\end{tabular}

In this work, the linear relation between the results from the DET gauge and those from the standard chemical analysis gave a slope of one Fig. (8), which indicates a good agreement between both results. Since $\mathrm{C}_{\mathrm{Al}}$ values as obtained by using the DET gauge were in good agreement with those obtained from the standard chemical analysis, we will conclude that the DET gauge is the best one for aluminum characterization. A slope with a slight shift far from 1 is obtained by the linear regression between the results from the SET gauge and those from the standard chemical analysis Fig. (7), which indicates some deviation between the nuclear and chemical results. Another reason for the preference of DET gauge on the SET gauge as an aluminum analyzer in this work is that the total error in $\mathrm{C}_{\mathrm{Al}}$ values in case of DET gauge is smaller than that in case of the SET gauge.

\section{Conclusion}

A nuclear gauge, based on single and dual energy $\gamma$-ray transmissions, has been developed and used to determine the concentration of the aluminum component in copper-aluminum alloy. The gauge is portable and uses a ${ }^{133} \mathrm{Ba} \gamma$ ray microsource as the main source radiation. The gauge does not require special shielding and does not expose the user to unacceptable levels of radiations. Aluminum concentration values with an accuracy of $98.53 \%$ and $99.09 \%$ have been obtained by using both single and $\gamma$-ray energy transmission techniques, respectively. In addition one can conclude the following:

1. For binary alloys, the most important criterion in this work is that the absorption coefficient of one of the material components should be much smaller than the absorption coefficient of the other one.

2. The present gauge has been successfully applied to binary alloys and it can be extended for the characterization of multiple component alloys with a dominated component. 
3. Although, the DET technique does not add any new to the SET technique, it still has the advantage of its independence on the target thicknesses, which enable us to avoid any errors due to thickness measurements.

\section{References}

1. R. A. M. Rizk, Nucl. Geophys., 3, 313 (1989).

2. B. D. Sowerby, Nucl. Geophys., 5, 491 (1991).

3. M.D. Rebgetz, J. S. Watt and H. W. Zastawny, Nucl. Geophys., 5, 497 (1991).

4. Y. Ma, L. Xiangdong and Y. Dazhan, Nucl. Sci. Tech., 4, 95 (1993).

5. A. Abedinzadeh, H. Rahimi, N. Rahimi, J. Moafian and Amini, Proceedings of a final research co-ordination meting, 145, 65 (1995).

6. R. A. M. Rizk, J, Rad. Nucl. Chem., 231, 25 (1997).

7. R. A. M. Rizk, A. H. El-kateb and A. M. Abdul-Kader J. Rad. Nucl. Chem., 242, 139 (1999).

8. B. Holynska, M. Lankosz and D. wegerzynek, IAEA/RL/139 (1987).

9. R. A. M. Rizk, J. Rad. Nucl. Chem., 231, 69 (1998).

10. P. Wyszomirski and J. Janczyszyn, Nucl. Geophys., 5(1/2), 53 (1991).

11. B. D. Sowerby, Int. J. Appl. Rad. Isot., 33, 1269 (1991).

12. J. S. Watt and J. Stefner, Int. J. Appl. Rad. Isot., 36, 867(1985).

13. M. J. Berger and J. H. Hubbell, NBSIR, 87, (1987). 